Article

\title{
Formulation Development of Albendazole-Loaded Self-Microemulsifying Chewable Tablets to Enhance Dissolution and Bioavailability
}

\author{
Somchai Sawatdee ${ }^{1,2, *}$, Apichart Atipairin ${ }^{1,2, *}$, Attawadee Sae Yoon ${ }^{1,2}$, Teerapol Srichana ${ }^{3}$, \\ Narumon Changsan ${ }^{4}$ and Tan Suwandecha ${ }^{5}$ \\ 1 Drug and Cosmetics Excellence Center, Walailak University, Thasala, Nakhon Si Thammarat 80161, \\ Thailand; attawadee.sa@wu.ac.th \\ 2 School of Pharmacy, Walailak University, Thasala, Nakhon Si Thammarat 80161, Thailand \\ 3 Drug Delivery System Excellence Center and Department of Pharmaceutical Technology, Faculty of \\ Pharmaceutical Sciences, Prince of Songkla University, Hat Yai, Songkhla 90112, Thailand; \\ teerapol.s@psu.ac.th \\ 4 Faculty of Pharmacy, Rangsit University, Pathumtani 12000, Thailand; narumon.c@rsu.ac.th \\ 5 Department of Pharmacology, Faculty of Sciences, Prince of Songkla University, Hat Yai, Songkhla 90112, \\ Thailand; tan.s@psu.ac.th \\ * Correspondence: somchai086@hotmail.com or somchai.sa@wu.ac.th (S.S.); apichart.at@wu.ac.th (A.A.); \\ Tel.: +66-7567-2818 (S.S.); +66-7567-2832 (A.A.); Fax: +66-7567-2814 (S.S.); +66-7567-2814 (A.A.)
}

Received: 20 February 2019; Accepted: 18 March 2019; Published: 20 March 2019

check for

\begin{abstract}
Albendazole is an anthelmintic agent with poor solubility and absorption. We developed a chewable tablet (200 mg drug equivalent), containing a self-microemulsifying drug delivery system (SMEDDS), with oral disintegrating properties. The emulsion was developed using sesame and soybean oils along with surfactant/co-surfactants, and the tablets were prepared by wet granulation using superdisintegrants and adsorbents. Infra-red (IR) spectral studies revealed no interaction between the drug and excipients, and all physical and chemical parameters were within acceptable limits. Stability studies for the formulation indicated no significant change over time. An in vitro release study indicated $100 \%$ drug release within $30 \mathrm{~min}$, and in vivo plasma concentrations indicated that the area under the curve (AUC) of albendazole in rats administered SMEDDS chewable tablets was significantly higher than in those administered commercial tablets or powder ( $p$-value $<0.05$ ). The systemic bioavailability of albendazole achieved through the SMEDDS tablets was 1.3 times higher than that achieved by the administration of comparable quantities of albendazole commercial tablets. This was due to the higher dissolution of albendazole SMEDDS in the chewable tablets. We conclude that the SMEDDS chewable formulation can be used to improve the dissolution and systemic availability of poorly water-soluble drugs.
\end{abstract}

Keywords: albendazole; self-microemulsion; chewable tablet; dissolution; bioavailability; pharmacokinetics

\section{Introduction}

Albendazole, or methyl(6-(propylthio)- $1 H$-benzoimidazol-2-yl) carbamate (Figure 1), a benzimidazole derivative, is a broad-spectrum anthelmintic agent with good efficacy in the treatment of echinococcosis, hydatid cysts, and neurocysticercosis caused by nematodes and cestodes [1-3]. It is poorly soluble, with an aqueous solubility of $0.2 \mu \mathrm{g} / \mathrm{mL}$ at $25^{\circ} \mathrm{C}, 1 \mu \mathrm{g} / \mathrm{mL}$ at $\mathrm{pH} 6.0$, and a $\log p$ value of 3.5. It has weak basic properties $\left(\mathrm{pK}_{\mathrm{a} 1}=2.68\right.$ and $\left.\mathrm{pK}_{\mathrm{a} 2}=11.83\right)[4,5]$. Albendazole falls into the biopharmaceutical classification system (BCS) class II category with a high permeability and low solubility. Because of its 
low aqueous solubility, it is poorly and erratically absorbed following oral administration. Following oral administration in rats, $20-30 \%$ is absorbed, and in humans, less than $5 \%$ is absorbed $[1,2,4,6]$.

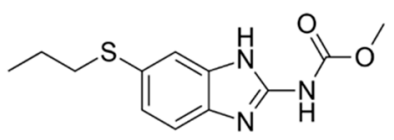

Figure 1. Chemical structure of albendazole.

To Improve the drug dissolution rate, several techniques have been developed and investigated such as particle size reduction, solid dispersion, inclusion complex formation, use of solubilizing agents, enhancement by surfactant systems, prodrug strategies and drug derivatization, lipid-based formulations, orally disintegrating tablets, self-emulsifying drug delivery system (SEDDS), and self-microemulsifying drug delivery system (SMEDDS) [7-9]. SMEDDS is a mixture of oils, surfactants and/or co-surfactant, one or more hydrophilic solvents and co-solvent [9-11]. Poorly water-soluble drugs can be dissolved in SMEDDS for oral administration. Upon contact with the aqueous phase of the GI tract, the digestive motility of the stomach and the intestine provide the necessary agitation for the spontaneous and fine dispersion of the SMEDDS formulations because the free energy required to form the emulsion is either low and positive or negative [12]. Although albendazole has been developed as SEDDS [4] and SMEDDS [13] to enhance the dissolution and bioavailability of drugs, liquid dosage forms are inconvenient to carry and difficult to administer as compared to solid dosage forms. In addition, solid pharmaceutical preparations are more stable than liquid preparations and their portability is convenient during the manufacturing process [14]. In fact, SMEDDS does not contain water in their composition, which enhances their chemical and physical stability. The major disadvantage of conventional SMEDDS is the high manufacturing cost as they have to be filled in soft gelatin capsules and they can interact with the shell components of the capsule in the SMEDDS. In addition, precipitation of either active ingredient and/or oil constituents can also be influenced by storage temperature [14,15]. Therefore, attention has been given to transform liquid into solid SMEDDS by several techniques such as spray drying, spray cooling, super critical fluid technology, and using adsorption carriers [14,16]. Colloidal silica, a successful inexpensive hydrophobic carrier, requires common laboratory instruments to formulate as a vehicle for the preparation of solid SMEDDS [16,17]. Carriers with absorbed SMEDDS are then compressed into tablets by wet granulation or direct compression methods [18,19]. However, they are difficult to formulate as tablets due to the high content of surfactant and oil in the formulation. There are no reports on the preparation of albendazole SMEDDS powder or tablets. Commercial albendazole tablets are available as chewable tablet dosage form $\left(\right.$ Zentel $\left.^{\mathrm{TM}}\right)$ in order to achieve rapid drug disintegration. The combination of albendazole SMEDDS with fast disintegrating strategies and their preparation as chewable tablets, similar to the market brand, are important for enhancing drug disintegration and improving dissolution of albendazole, thereby increasing bioavailability.

In this study, we developed a new self-microemulsifying formulation of albendazole combining an orally disintegrating system as a chewable tablet to enhance the dissolution rate of albendazole. We investigated the oral bioavailability of this formulation in rats in comparison with conventional albendazole tablets and powder.

\section{Materials and Methods}

\subsection{Solubility Studies}

An albendazole solubility experiment was carried out according to previous report [4]. Stock solution of albendazole $500 \mu \mathrm{g} / \mathrm{mL}$ in dimethyl sulfoxide (DMSO) was spike in to various oils, surfactants, and co-surfactants by using a 96-well plate format. The 96-well plate was shaken for $2 \mathrm{~h}$ and then centrifuged at $4000 \mathrm{rpm}$ at $37^{\circ} \mathrm{C}$ on a Sigma centrifuge (Sigma Laborzentrifugen 
$\mathrm{GmbH}$, Osterode am Harz, Germany) for $10 \mathrm{~min}$. The supernatant was used for high-performance liquid chromatography (HPLC) analysis according to method described in following section.

\subsection{Construction of Pseudo-Ternary Phase Diagram}

Pseudo-ternary phase diagrams were used for the selection of microemulsion area using water titration method. The pseudo-ternary phase diagrams consisting of water, oil, surfactant/co-surfactant mixture of different hydrophilic-lipophilic balance (HLB) values were constructed at room temperature $\left(25 \pm 5{ }^{\circ} \mathrm{C}\right)$. The oils employed were sesame oil (Namsiang Co. Ltd., Bangkok, Thailand), soybean oil (Thanakorn Vegetable Oil Products Co., Ltd., Samutprakan, Thailand), Captex 300 Low C6 and Capmul PG8 (medium-chain triglycerides and propylene glycol monocaprylate, respectively, both from Abitec Corporation, Columbus, OH, USA) and Labrafac Lipofile WL1349 (Gattefosseé, SA, France). Surfactant and co-surfactant were Tween80 (Namsiang Co. Ltd., Bangkok, Thailand), Solutol HS 15 (poly-oxyethylene esters of 12-hydroxystearic acid; Sigma-Aldrich, St. Louis, MO, USA), Cremophor RH40 (PEG 400 hydrogenated castor oil, BASF, Washington, NJ, USA) and propylene glycol (S. Tong Chemical Co., Ltd., Nonthaburi, Thailand). The ratio of surfactant and co-surfactant was fixed at $1: 1,1: 2,1: 3,3: 1$ and $2: 1$ on the mass ratio. The mixtures of surfactant and co-surfactant with water were prepared at ratios of 10:0, 9:1, 8:2, 7:3, 6:4, 5:5, 4:6, 3:7, 2:8, 1:9, and 0:10 (w/w). Each mixture of oil and surfactant/co-surfactant was titrated with water and visually observed for phase clarity and flowability. The titration endpoint was defined as the point where the mixture became turbid and phase separation was observed. The resulting mixtures were identified as microemulsions when they appear monophasic, transparent or translucent and easily flowable liquid with low viscosity. The microemulsion regions are indicated on the ternary graph.

\subsection{Preparation of Albendazole Self-Microemulsifying Drug Delivery System (SMEDDS)}

After a series of self-microemulsion formulations was selected from the ternary diagram of surfactants, co-surfactants, and oils, the solubility of albendazole was carried out by HPLC assay. The microemulsion system made with the highest solubility of albendazole was selected to prepare albendazole SMEDDS. An albendazole SMEDDS were prepared as a stock solution at a concentration $0.4 \% w / w$ and stored at room temperature until further use. Briefly, albendazole was accurately weighed, then placed in a beaker, dispersed into an oil phase, and heated at $40-50{ }^{\circ} \mathrm{C}$ under vortex. Surfactant and co-surfactant were mixed together in a separate test tube and mixed well under vortex, then heated to $60^{\circ} \mathrm{C}$ to mix properly. The drug-containing oil phase was transferred into the surfactant and co-surfactant mixture under continuous mixing, vortexed and heated at $50{ }^{\circ} \mathrm{C}$ in a sonicator until albendazole was completely dissolved.

\subsection{Emulsion Droplet Size Measurement}

The particle size measurement was carried out by using a Malvern Zetasizer (Worcestershire, UK) equipped with $2000 \mathrm{Hydro} \mathrm{MU}$ at $25^{\circ} \mathrm{C}$. The particle size measurement was in a range 0f $0.02-2000$ $\mu \mathrm{m}$. Each microemulsion was aliquot of $500 \mu \mathrm{L}$ and diluted to $250 \mathrm{~mL}$ with Milli-Q water in a beaker using a magnetic stirrer. The resultant emulsion was analysis and particle size was calculated based on volume size distribution.

\subsection{Formulation Development of Albendazole SMEDDS Chewable Tablets}

Albendazole SMEDDS chewable tablets were prepared using an optimized microemulsion system based on the procedures described above, with a target dose of $200 \mathrm{mg}$. Albendazole was dissolved in the microemulsion system at a concentration of about $0.4 \mathrm{mg} / \mathrm{mL}$, corresponding to an albendazole dose of $40 \mathrm{mg}$. $160 \mathrm{mg}$ of albendazole powder was further added to prepare the SMEDDS chewable tablets.

To prepare 1000 tablets, albendazole $40 \mathrm{~g}$ was dissolved in a microemulsion system at $100 \mathrm{~mL}$. This was vortex mixed and heated at $50{ }^{\circ} \mathrm{C}$ in a sonicator until albendazole dissolved. Albendazole 
powder and all excipients were passed through a No. 40 sieve before use. Albendazole powder $160 \mathrm{~g}$ was mixed with dried lactose monohydrate (P.C. Drug Center Co., Ltd., Bangkok, Thailand), maltodextrin (Brentag Ingredients Public Co. Ltd., Bangkok, Thailand), milk powder and cocoa powder (Cocoa Dutch ${ }^{\circledR} 100 \%$ instant cocoa), sucrose (Mitr Phol ${ }^{\circledR}$ pure refined sugar), and sodium starch glycolate (P.C. Drug Center Co., Ltd., Bangkok, Thailand) until homogeneous. Albendazole microemulsion was then absorbed in colloidal silicon dioxide (P.C. Drug Center Co., Ltd., Bangkok, Thailand), maltodextrin, and mannitol (P.C. Drug Center Co., Ltd., Bangkok, Thailand) (1:1:1 ratio by weight) and added to albendazole mixture powders using a planetary kneader at a mixing speed of $100 \mathrm{rpm}$ until a homogeneous paste formed. This was dried in a vacuum oven at $70{ }^{\circ} \mathrm{C}$ for $12 \mathrm{~h}$ to keep the moisture content below $2 \%$. The dried granules were then passed through a No. 14 sieve. Sodium starch glycolate was added as an extragranular disintegrant and vanilla powder and lactose monohydrate were added as diluents (all obtained from P.C. Drug Center Co., Ltd., Bangkok, Thailand) to maintain an equivalent weight.

Magnesium stearate (P.C. Drug Center Co., Ltd., Bangkok, Thailand) was used as a tablet lubricant and was passed through a No. 60 sieve before use. Albendazole SMEDDS granules were blended with sodium starch glycolate used as a superdisintegrant for $5 \mathrm{~min}$ in a plastic bottle, then further blended with sieved magnesium stearate (P.C. Drug Center Co., Ltd., Bangkok, Thailand) for $3 \mathrm{~min}$ in the same bottle. The final mixtures were compressed into $700 \mathrm{mg}$ tablets using a single punch tableting machine (small tablet press machine Model SP-KR, Charatchai machinery, Thailand) with a round punch and a die diameter of $12 \mathrm{~mm}$. The compression forces $(50 \mathrm{kN})$ were kept constant in order to compare other properties.

Physical mixing formulation without the granulating process (F3) was prepared in a similar manner to serve as controls. Briefly, albendazole and all excipients were mixed and compressed powder into a tablet as F3. This formulation was prepared by direct compression process without granulating solvent. Formulation without the albendazole microemulsion (F4) were prepared by mixing albendazole $200 \mathrm{mg} /$ tablet with other excipients excluding the microemulsion system, with purified water added as a granulating solvent and mixed to obtain wet mass. Lactose monohydrate was used in this formulation in place of the microemulsion system. The damp mass was sieved and dried in a hot air oven $\left(70^{\circ} \mathrm{C}\right)$ and passed through a No. 14 sieve, mixed with an extragranular excipient, and compressed into tablets. A schematic diagram of this process is shown in Figure 2, and the details of each formula are given in Table 1.

Table 1. Composition of albendazole self-microemulsion chewable tablet formulations.

\begin{tabular}{ccccc}
\hline \multirow{2}{*}{ Ingredients (mg/tablet) } & \multicolumn{4}{c}{ Formulation Codes } \\
\cline { 2 - 5 } & F1 & F2 & F3 & F4 \\
\hline Albendazole (in microemulsion) & 40 & 40 & - & - \\
Soybean oil & - & 34 & 34 & - \\
Sesame oil & 24 & - & - & - \\
Cremophor RH40 & - & 33 & 33 & - \\
Polyethylene glycol (PEG) 400 & 38 & - & - & \\
Tween80 & 38 & 33 & 33 & - \\
Albendazole (as powder) & 160 & 160 & 200 & 200 \\
Maltodextrin & 70 & 70 & 70 & 70 \\
Mannitol & 70 & 70 & 70 & 70 \\
Colloidal silicon dioxide & 70 & 70 & 70 & 70 \\
Lactose monohydrate & 20 & 20 & 20 & 120 \\
Sucrose & 25 & 25 & 25 & 25 \\
Sodium starch glycolate & 28 & 28 & 28 & 28 \\
Milk powder & 60 & 60 & 60 & 60 \\
Coco powder & 50 & 50 & 50 & 50 \\
Vanilla powder & 0.1 & 0.1 & 0.1 & 0.1 \\
Magnesium stearate & 7 & 7 & 7 & 7 \\
Purified water * & 0.2 & 0.2 & - & 0.2 \\
Total weight & 700 & 700 & 700 & 700 \\
\hline * evaporated during manufacturing. &
\end{tabular}



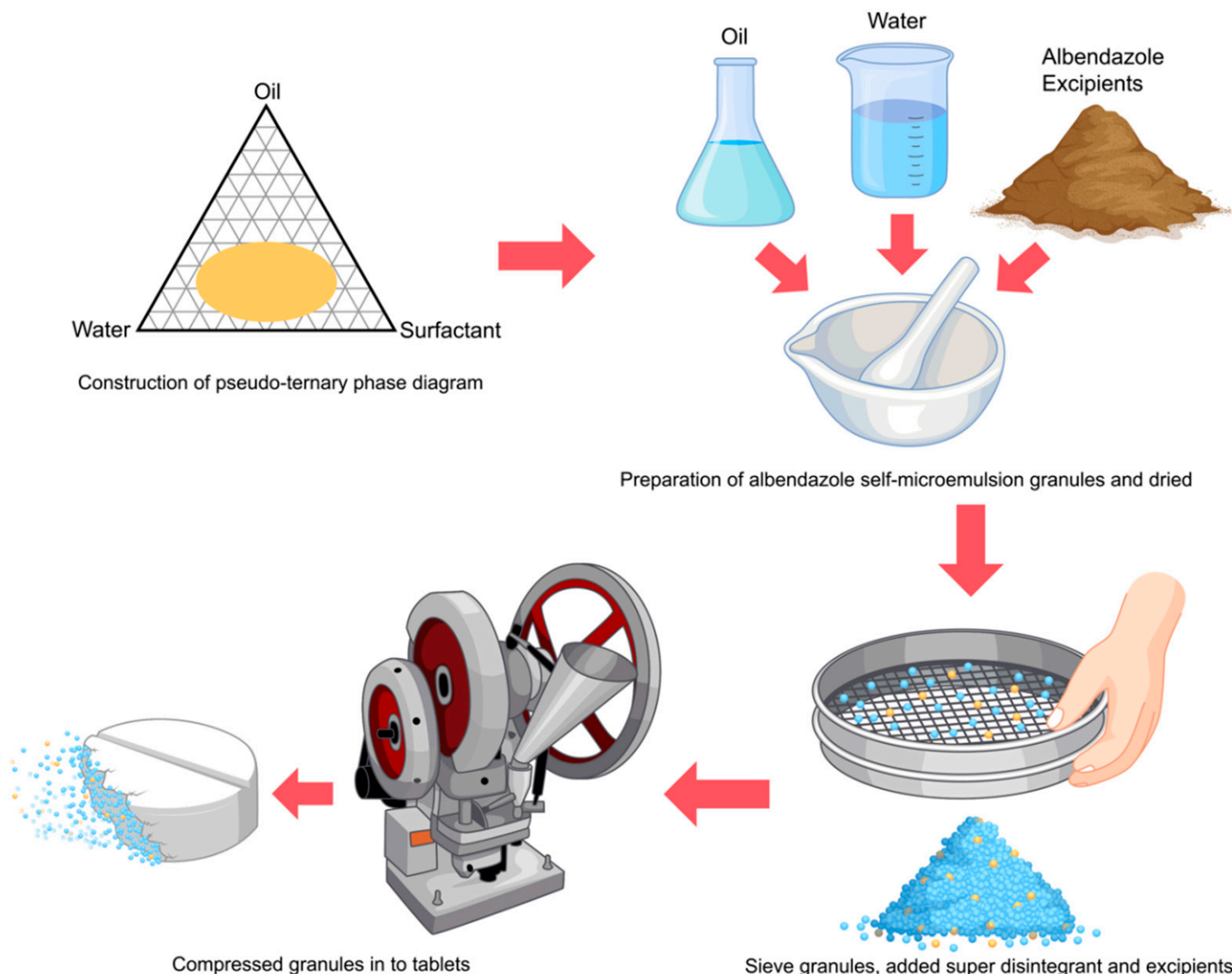

Compressed granules in to tablets

Sieve granules, added super disintegrant and excipients

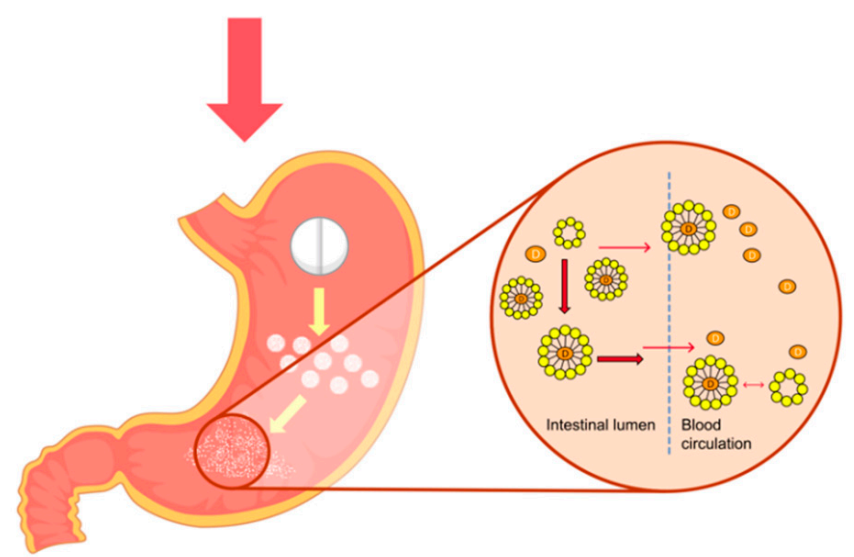

Schematic diagram of albendazole self-microemulsion tablets dissolved in gastrointestinal tract

Figure 2. Schematic diagram of the method preparation of albendazole self-microemulsifying drug delivery system (SMEDDS) chewable tablets with a proposed dissolution mechanism enhancing bioavailability.

\subsection{Analysis of Albendazole by High-Performance Liquid Chromatography (HPLC)}

The concentrations of albendazole and albendazole sulfoxide in the formulations and in plasma samples were determined using HPLC as previously reported with a modification [4,20-22]. Method validation (specificity, linearity, precision, accuracy, limit of detection (LOD), and limit of quantitation (LOQ)) was performed before analysis. In addition, the system's suitability parameter was also calculated. The HPLC instrument (Ultimate 3000, Thermo Fisher Scientific, Dionex Corporation, Sunnyvale, CA, USA) equipped with quaternary pump, degasser, a sample loop with an injection volume of $20 \mu \mathrm{L}$, and an autosampler. Data was recorded using Chromeleon 7 software. Separations 
were performed on a $250 \mathrm{~mm}$ long $\times 4.6 \mathrm{~mm}$ internal diameter reversed-phase stainless steel column (Inertsil ${ }^{\circledR}$ ODS-3, GL Sciences Inc., Japan) filled with $5 \mu \mathrm{m}$ octadecylsilane and maintained at $25^{\circ} \mathrm{C}$. The mobile phase consisted of a degassed mixture of hexane and ethanol in a ratio of 89:11 by volume at ambient temperature. The flow rate was maintained at $1.0 \mathrm{~mL} / \mathrm{min}$, and separation was monitored by ultraviolet (UV) detection at a wavelength of $291 \mathrm{~nm}$. The calibration curve was found to be linear in a range of $0.02-4 \mu \mathrm{g} / \mathrm{mL}$.

\subsection{Powder X-ray Diffraction (PXRD)}

Diffractograms of F1-F4 were developed using a powder X-ray diffractometer (Rigaku RU200, Rigaku Corp., Tokyo, Japan). The measuring conditions were as follows: graphite-monochromated $\mathrm{Cu}$ $\mathrm{K} \alpha$ radiation; voltage $40 \mathrm{kV}, 300 \mathrm{~mA}$ and angle speed of $4{ }^{\circ} \mathrm{C} / \mathrm{min}$ over the range of $5-45^{\circ}$.

\subsection{Fourier Transform Infrared Spectroscopy (FT-IR)}

A small amount of albendazole raw material, albendazole SMEDDS chewable tablets (F1, F2), control tablet formulation (F3, F4) and excipients were grinding mixed into KBr pellets in a small mortar and pestle. The sample with $\mathrm{KBr}$ mixture paste was compressed into tablets using a hydraulic press prior to measurement of the infrared (IR) spectrum at ambient temperature. The functional groups of albendazole, albendazole formulations and excipients were recorded in the frequency range of 4000-400 $\mathrm{cm}^{-1}$ using a Fourier transform infrared (FT-IR) spectrophotometer (Perkin Elmer Inc., Waltham, MA, USA).

\subsection{Physical and Mechanical Properties of Granules and Tablets}

\subsubsection{Angle of Repose}

Granule or powder formulations were each placed in a funnel hung at a fixed height using a burette stand and allowed to fall onto a graph paper, forming a heap. The height and the radius of the heap was measured and the angle of repose was calculated using the formula given in Equations (1) or (2).

$$
\tan \theta=\frac{\text { height of the heap formed }(\mathrm{h})}{\text { radius of the heap }(\mathrm{r})},
$$

or

$$
\theta=\tan ^{-1} \mathrm{~h} / \mathrm{r}
$$

\subsubsection{Hardness Test}

The hardness of the F1-F4 tablets was measured using a PTB311E model (Pharma Test, Hainburg, Germany) and expressed as a mean value standard deviation.

\subsubsection{Thickness Test}

The dimensions of F1-F4 tablets were measured using a Vernier caliper. Six measurements were taken and expressed as a mean value \pm standard deviation.

\subsubsection{Friability Test}

Ten tablets of each category were accurately weighed and placed in a plastic chambered friability apparatus (Erweka, model TA220, Heusenstamm, Germany) described in USP36 [23]. The chamber was attached to a motor revolving at $25 \mathrm{rpm}$ for $4 \mathrm{~min}$. The tablets were weighed again, and the percentage weight loss (friability) was calculated using the following formula:

$$
\text { Friability }=\frac{\text { Initial weight }- \text { Final weight }}{\text { Initial weight }} \times 100
$$




\subsubsection{Disintegration Test}

The disintegration test for the tablets was performed using a disintegration apparatus with discs (Pharma Test, model DIST3, Hainburg, Germany) described in USP36 [23] with six replicates for each tablet group. Tablets were placed individually in each tube in a $900 \mathrm{~mL}$ beaker of distilled water maintained at a temperature of $37 \pm 2{ }^{\circ} \mathrm{C}$. The average values of the disintegration time and standard deviations were calculated.

\subsection{Content of Albendazole SMEDDS Chewable Tablets}

Ten tablets of selected formulation were weighed and grounded to a fine powder, and a quantity equivalent to $200 \mathrm{mg}$ albendazole (700 $\mathrm{mg}$ of powder) was introduced into a $100 \mathrm{~mL}$ volumetric flask and diluted with the mobile phase. The solution obtained was sonicated for $15 \mathrm{~min}$ and filtered through a $0.45 \mu \mathrm{m}$ nylon membrane filter. The filtrate was suitably diluted with the mobile phase prior to HPLC analysis as previously described. The mean percentage of the drug content was determined based on three replicates.

\subsection{In Vitro Dissolution Studies}

A United State Pharmacopoeia dissolution apparatus II (Varian, Vankel VK7010, Palo Alto, CA, USA) with paddle rotation speed of $50 \mathrm{rpm}$ was used to monitor the in vitro dissolution profiles of albendazole. The dissolution values of albendazole self-microemulsion chewable tablets (F1 and F2) were compared to the albendazole control formulation by physical mixing method (F3), conventional wet granulation without SMEDDS excipient (F4) and a commercial product which was purchased from a drug store in Thailand. The dissolution profile was performed in $900 \mathrm{~mL} 0.1 \mathrm{M} \mathrm{HCl}$ as the dissolution medium described in several research works at $37 \pm 0.5^{\circ} \mathrm{C}$ [23-26]. During the study, $5 \mathrm{~mL}$ aliquots were taken at predetermined time intervals from the dissolution medium and $5 \mathrm{~mL}$ of fresh medium were replaced. Samples were withdrawn from the dissolution vessels at $0,0.25,0.5$, $0.75,1,1.5,2,2.5,3,3.5,4,4.5,5,10,20$, and $30 \mathrm{~min}$ and passed through a $0.45 \mu \mathrm{m}$ nylon membrane filter prior to analysis. The amount of albendazole was determined using HPLC as described above. The dissolution experiments were carried out in triplicate.

\subsection{Stability Studies}

Selected formulations of the albendazole SMEDDS chewable tablets were packed in Alu-PVC blister packaging covered with aluminum foil, in order to block moisture and light, and kept under accelerated conditions $\left(40{ }^{\circ} \mathrm{C} / 75 \%\right.$ relative humidity $\left.(\mathrm{RH})\right)$, long-term stability conditions $\left(30^{\circ} \mathrm{C} / 75 \%\right.$ $\mathrm{RH})$, and at room temperature with ambient relative humidity following the Association of Southeast Asian Nations Guidelines the Stability of Drug Products according to climatic zone IVb $[27,28]$. Tablets were evaluated for appearance, disintegration time, and drug content over periods of 1 , 3 , and 6 months.

\subsection{Animals}

Male Wistar rats were purchased from the National Laboratory Animal Center, Mahidol University, Thailand. Animals were fed until weighting about 250-300 g before used. All animals had free access to pelleted food and tap water ad libitum prior to the experiments, and were housed in clean polypropylene or corrugated paper cages. Temperature was maintained at controlled room temperature $\left(25 \pm 2{ }^{\circ} \mathrm{C}\right)$ and humidity of $50-60 \%$ with a $12 \mathrm{~h}$ light and dark cycle throughout the experiment. These experimental procedures were approved by the Animal Ethical Committee, Walailak University, Nakhon Si Thammarat, Thailand (approval no. 004/2559) before performed the experiment. 


\subsection{Drug Administration and Sampling}

Overnight fasted rats were divided into three groups with five rats in each. Albendazole SMEDDS chewable tablets, commercial albendazole tablets (Zentel ${ }^{\mathrm{TM}}$ ), or albendazole raw material were administered orally as a single dose (p.o.) using a gastric gavage tube to rats in each group. The selected formulation tablets (F2) or Zentel ${ }^{\mathrm{TM}}$ tablets were crushed to a fine powder by mortar and pestle. The fine tablets powder ( $\mathrm{F} 2$ or Zentel ${ }^{\mathrm{TM}}$ ) and albendazole raw material were weight equivalent to $50 \mathrm{mg}$ per $\mathrm{kg}$ of each animal weight. The drug powders were each dispersed in $2 \mathrm{~mL}$ of distilled water and mixed homogeneously prior to oral administration. Blood samples $(0.5 \mathrm{~mL})$ were collected via the tail artery at $0,15,30,60,90,120,180$, and $240 \mathrm{~min}$ after the oral administration of albendazole. The samples were immediately transferred to a heparinized microcentrifuge tube and centrifuged at $4000 \mathrm{~g}$ for $20 \mathrm{~min}$ at $4{ }^{\circ} \mathrm{C}$. Plasma samples were removed to Eppendorf tubes for further use. During the study, the animals received water ad libitum.

\subsection{Preparation of Samples}

Each $400 \mu \mathrm{L}$ of separated plasma sample was immediately mixed with $2 \mathrm{~mL}$ methanol in a vortex mixer for $15 \mathrm{~s}$ and centrifuged at $2000 \mathrm{rpm}$ for $5 \mathrm{~min}$. The supernatant was collected and stored at $-70{ }^{\circ} \mathrm{C}$ prior to use. The procedures used for sample preparation and handling were done within $24 \mathrm{~h}$ of blood sample collection. The supernatant was transferred to another Eppendorf tube for HPLC analysis [20].

\subsection{Pharmacokinetics and Statistical Analysis of Data}

After treatment in each animal, the albendazole sulfoxide concentration versus time curves obtained from individual animals were fitted using WinNonlin software version 5.2 (Pharsight Corp, Mountain View, Sunnyvale, CA, USA) and reported as mean \pm standard deviation (SD). The pharmacokinetic parameters for each animal were analyzed via non-compartmental model analysis for three formulations (albendazole SMEDDS chewable tablets, albendazole tablets commercial product, and albendazole powder). The pharmacokinetic parameters including the area under the concentration-time curve $\left(\mathrm{AUC}_{0-\infty}\right)$, maximum concentration $\left(C_{\max }\right)$, time to reach maximum concentration ( $\left.T_{\max }\right)$ and half-life $\left(t_{1 / 2}\right)$ were determined by trapezoidal rule. The relative bioavailability $(\mathrm{F})$ was calculated according to the following equation:

$$
\mathrm{F}=\frac{\mathrm{AUC}(\text { test formulation })}{\mathrm{AUC}(\text { reference })}
$$

where AUC of the reference is the AUC of the albendazole commercial product group and AUC test formulation is the AUC of albendazole SMEDDS chewable tablet or albendazole powder.

The pharmacokinetic parameters were reported as mean \pm SD. Pharmacokinetic parameters were statistically compared using a one-way analysis of variance (ANOVA). Mean values were considered significantly different at $p<0.05$.

\section{Results and Discussion}

\subsection{Construction of a Pseudo-Ternary Phase Diagram}

SMEDDS are a mixture of oil, surfactant, and co-surfactant with a drug dissolved in the system to improve the absorption of poorly water-soluble drugs. These systems form a microemulsion when exposed to an aqueous phase in the GI tract under mild agitation. Therefore, to select the SMEDDS composition for albendazole, its solubility in various vehicles was studied. The results indicated that sesame oil, soybean oil, Capmul PG8, Labrafac Lipophile WL1349, Cremophor RH40, Tween80, and PEG400 were the most effective in developing solvent mixtures because of the high solubility of 
albendazole (data not shown) in these vehicles. Therefore, we used these ingredients to construct a pseudo-ternary phase diagram.

The construction of a pseudo-ternary phase diagram in the absence of active ingredient was used to identify the optimized concentrations of oil, surfactant and co-surfactant in the liquid SMEDDS formulation. Their self-emulsification properties were visually observed after SMEDDS were prepared $[17,29,30]$. Diagrams of oil (sesame oil, soybean oil, Capmul PG8, and Librafac Lipophile WL1349), surfactants and co-surfactants (Cremophor RH40, Tween80, and PEG 400), and water are provided in Figure 3. A large microemulsion region obtained from sesame oil with PEG400 and Tween 80, in a 2:1 ratio, in water yielded a clear and transparent solution (Figure 3C). A second large microemulsion region was prepared using soybean oil with Cremophor RH40 and Tween 80 in a ratio of 2:1 (Figure 3B). The microemulsion containing 20\% sesame oil, 40\% PEG400:Tween 80 (2:1), and 40\% water is shown in Figure 4. Sesame and soybean oils have been developed as self-nanoemulsifying drug-delivery systems due to their solubilizing capability and low toxicity [31,32]. Thus, sesame oil and soybean oil were selected as oil phases for the preparation of albendazole-loaded SMEDDS containing chewable tablets in the next step. Cremophor RH40 (PEG-40 hydrogenated castor oil and HLB 14-16) and PEG400 were used as the surfactant and co-surfactant, respectively. Cremophor RH40 is widely used as an emulsifying and solubilizing agent especially for albendazole [4]. Generally, non-ionic surfactants are considered less toxic than ionic surfactants, and are usually accepted for oral ingestion [33,34]. PEG400 and Tween 80 also enhanced drug solubility, and when mixed with Cremophor RH40, yielded a suitable HLB value and viscosity to form a fine microemulsion. Furthermore, PEG400 and Tween80 are powerful solubilizing agents used in several dosage forms [32]. Based on these results, the compositions of sesame oil, soybean oil, PEG 400, Tween80, and Cremophor RH40 were selected for preparing self-microemulsions of albendazole granules.

Based on the pseudo-ternary phase diagram, soybean oil with Cremophor RH40/Tween80 and sesame oil with PEG400/Tween80 in water (Figure 3B,C) showed a large microemulsion region. The $\mathrm{z}$-average particle size of the liquid and solid SMEDDS were assessed. From Figure 3B,C, in the soybean oil or sesame oil/PEG400 or Cremophor RH40/Tween80 system, we observed that the liquid SMEDDS formulation of $20 \%$ of soybean oil or sesame oil, $30 \%$ Cremophor RH40, and $50 \%$ Tween 80 showed the smallest z-average diameter (around $150 \mathrm{~nm}$ for both systems) (Table 2). Therefore, this composition was used as an optimal liquid SMEDDS. Furthermore, $10 \%(w / w)$ of the drug was entirely dissolved in this formulation and particle size larger than liquid SMEDDS without the drug but these were not significantly different. Moreover, the z-average particle sizes of other liquid SMEDDS system (Figure 3A,D-F) were similar.

The assessment of self-emulsification can be carried out by visually observing the emulsification and droplet formation of the liquid SMEDDS formulations. Spontaneous emulsion formation was not efficient when the volume of surfactant was less than that of the oil in liquid SMEDDS. In the case of sesame oil/PEG400/Tween80 system, the efficiency of emulsification was good when the concentration of the surfactant/co-surfactant was more than $60 \% v / v$ of the liquid SMEDDS formulation. Similarly, in the case of soybean oil/Cremophor RH40/Tween80 system, the efficiency of emulsification was good when the concentration of the surfactant/co-surfactant was more than $70 \% v / v$ of the liquid SMEDDS formulation. 
(A)

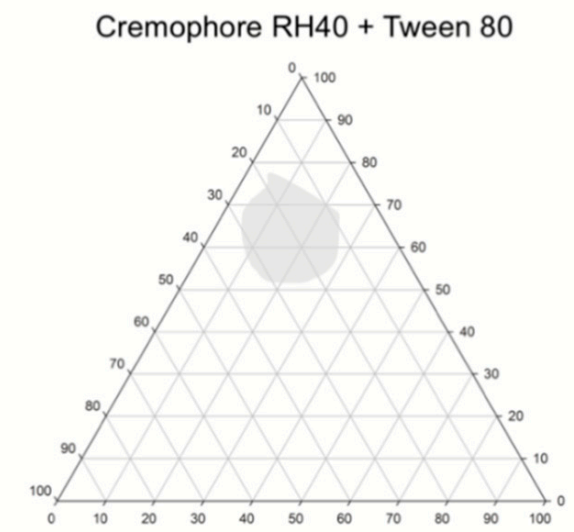

Sesame oil
(B)

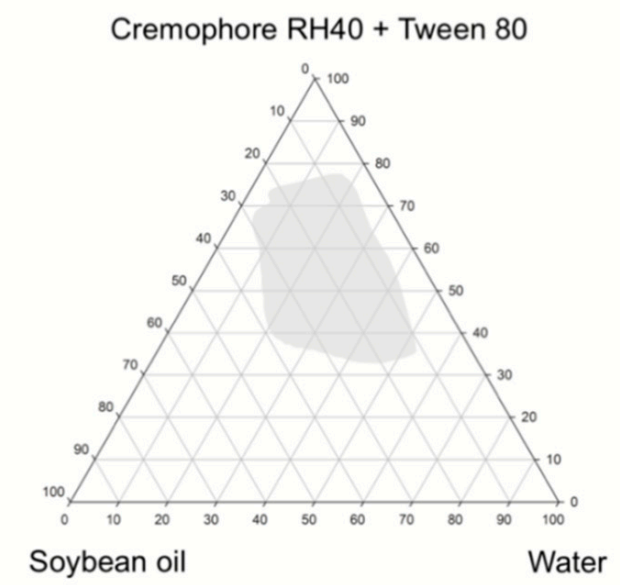

(D)

(C)

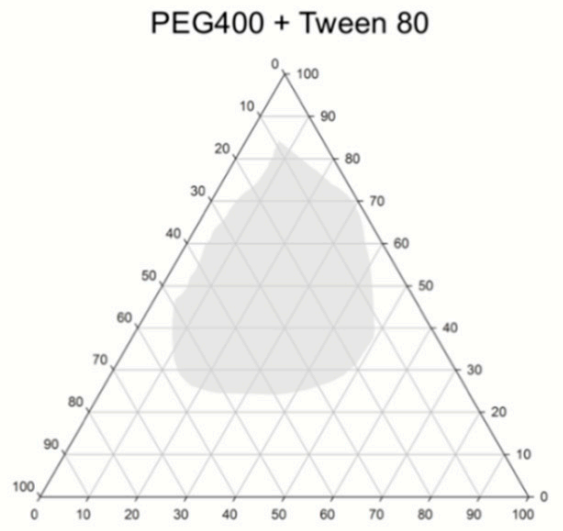

Sesame oil

Water

(E)

Cremophore $\mathrm{RH} 40+$ Tween 80

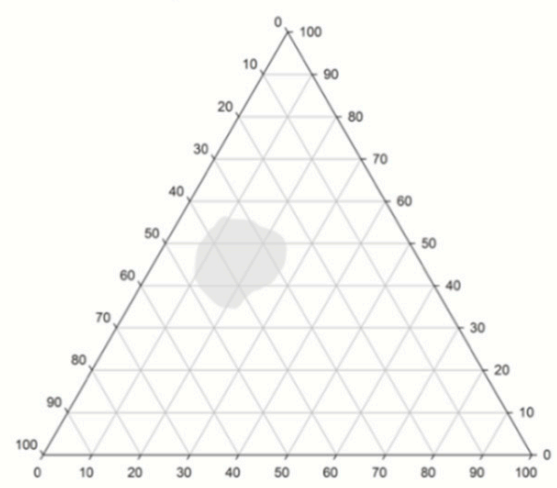

Capmul PG8

Water

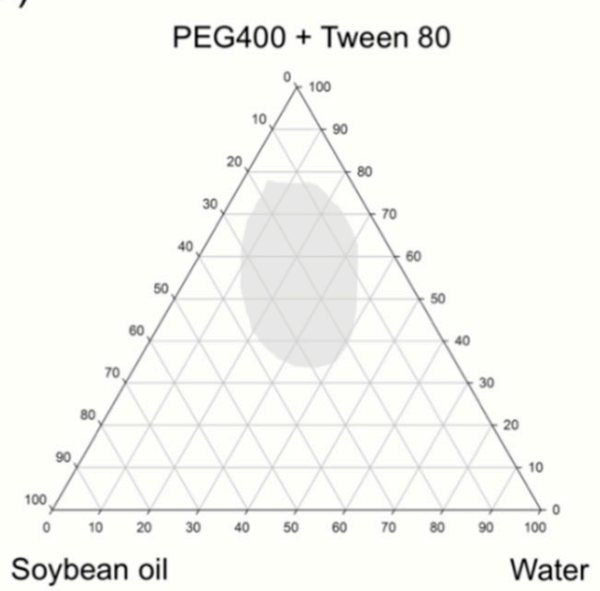

(F)

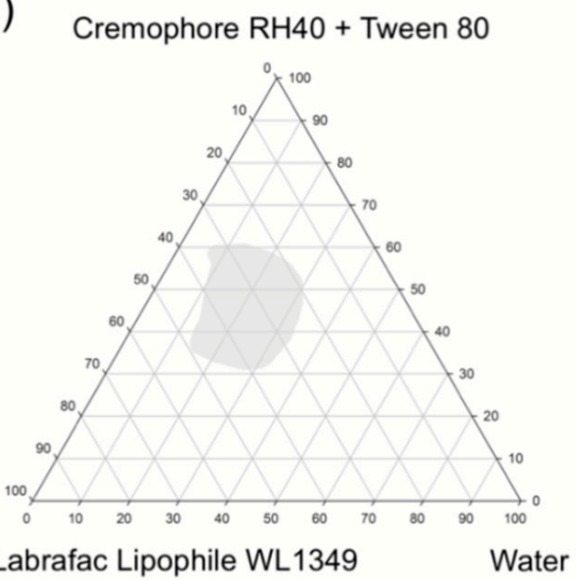

Figure 3. Pseudo-ternary phase diagram of various surfactant, co-surfactant, oil, and water. Microemulsion regions of the ternary plot are indicated in the gray areas. 


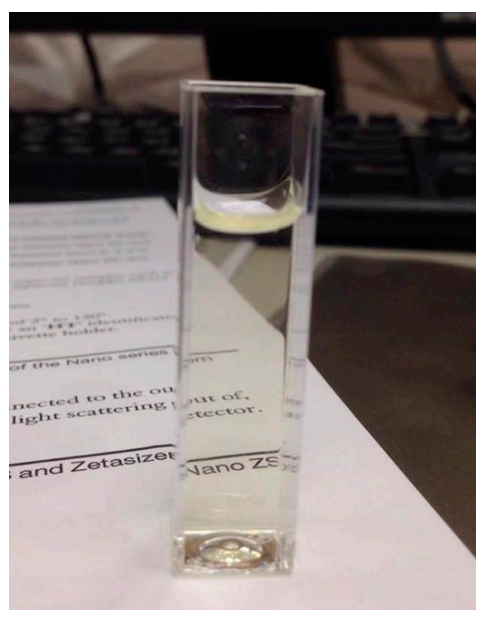

Figure 4. Appearance of microemulsion containing sesame oil, PEG400, Tween80 and water.

Table 2. Particle size of liquid SMEDDS with and without $10 \%$ albendazole (mean \pm SD, $n=3$ ).

\begin{tabular}{ccc}
\hline Liquid SMEDDS & Particle Size (nm) & Polydispersity index (PDI) \\
\hline $20 \%$ Sesame oil & & \\
$30 \%$ PEG400 & $152.5 \pm 10.1$ & $0.234 \pm 0.005$ \\
$50 \%$ Tween 80 & & \\
without albendazole & & \\
\hline $20 \%$ Sesame oil & $168.9 \pm 10.3$ & \\
$30 \%$ PEG400 & & \\
$50 \%$ Tween 80 & & \\
\hline with $10 \%$ albendazole dissolved & $149.9 \pm 12.3$ & \\
\hline $20 \%$ Soybean oil & & \\
$30 \%$ Cremophor RH40 & & \\
$50 \%$ Tween 80 & & \\
without albendazole & $159.9 \pm 19.1$ & \\
\hline $20 \%$ Soybean oil & & \\
$30 \%$ Cremophor RH40 & & \\
$50 \%$ Tween 80 & & \\
with $10 \%$ albendazole dissolved & & \\
\hline
\end{tabular}

\subsection{Preparation of Albendazole Self-Microemulsion Chewable Tablets}

Solid carriers are mainly divided into either water-soluble carriers or water-insoluble carriers [35,36]. Colloidal silicon dioxide is non-porous silica with hydrophobic properties. It is used as a water-insoluble carrier and approximately $1 \mathrm{~g}$ of colloidal silicon dioxide can be used to solidify $1 \mathrm{~g}$ of SMEDDS [35,36]. Water-insoluble carriers have high oil-adsorbing capacity and thus minimize the amount required to solidify the SMEDDS, but an incomplete desorption of SMEDDS components can occur because of hydrophobic interactions between the drug and water-insoluble solids [36-38]. Mannitol is a non-hygroscopic isomer of sorbitol and also used as a solidifying carrier [36,39,40]. Maltodextrin is a polysaccharide and widely used as a tablet excipient to improve tablet properties such as liquid absorbance and increased porosity with rapidly absorbing capacity [41-43]. Only colloidal silicon dioxide is enough for absorbing liquid SMEDDS with albendazole. Thus, in this study, maltodextrin and mannitol were used as tablet excipients while formulating the chewable tablets. However, other absorbents can be for absorption during the granule preparation. In this study, we selected sodium starch glycolate as a disintegrant due to its fast-disintegrating properties. Other ingredients including milk powder, cocoa powder, sugar, and vanilla flavor powder were chosen for their good taste in chewable tablets. In addition, we selected lactose for solidification, because of its popularity as a diluent in commercial products. 
Since about $40 \mathrm{mg}$ albendazole was dissolved in the self-microemulsion system, albendazole powder was added to the formulation at $160 \mathrm{mg}$ per tablet (Table 3). Figure $5 \mathrm{~A}$ shows the appearance of the albendazole self-microemulsifying powder after the absorption of colloidal silicon dioxide in it, and Figure 5B depicts the appearance after the absorbed albendazole was formulated as a granule by adding tablet excipients, prior to tableting.

Table 3. Solubility of albendazole in selected self-microemulsifying formulations.

\begin{tabular}{ccccccc}
\hline \multirow{2}{*}{ Composition } & \multicolumn{7}{c}{ Formulation (\%) } \\
\cline { 2 - 7 } & A & B & C & D & E & F \\
\hline Sesame oil & 34 & - & 24 & - & - & - \\
Soybean oil & - & 34 & - & 32 & - & - \\
Capmul PG8 & - & - & - & - & 30 & - \\
Labrafac Lipophile & - & - & - & - & - & 35 \\
WL1349 & 33 & 33 & - & 45 & 27 & 44 \\
Cremophor RH40 & 33 & 33 & 38 & 23 & 43 & - \\
Tween80 & - & - & 38 & - & - & $0.28 \pm 0.17$ \\
PEG400 & $0.38 \pm 0.02$ & $0.41 \pm 0.01$ & $0.43 \pm 0.02$ & $0.39 \pm 0.02$ & $0.16 \pm 0.04$ & 0.28 \\
\hline Solubility of & & & & & & \\
albendazole $(\mathrm{mg} / \mathrm{mL})$ & &
\end{tabular}

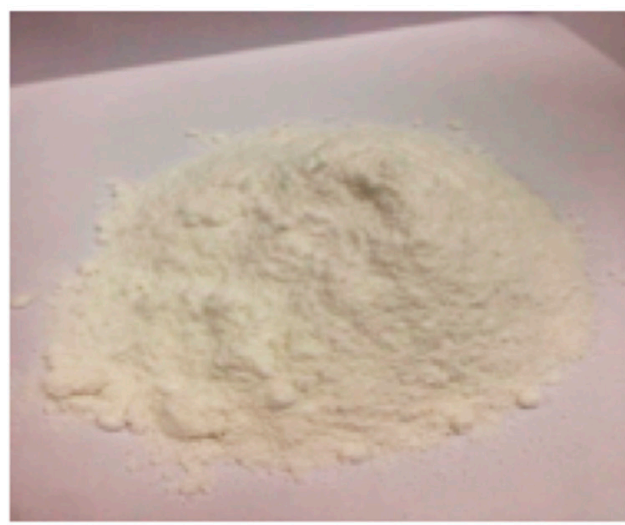

(A)

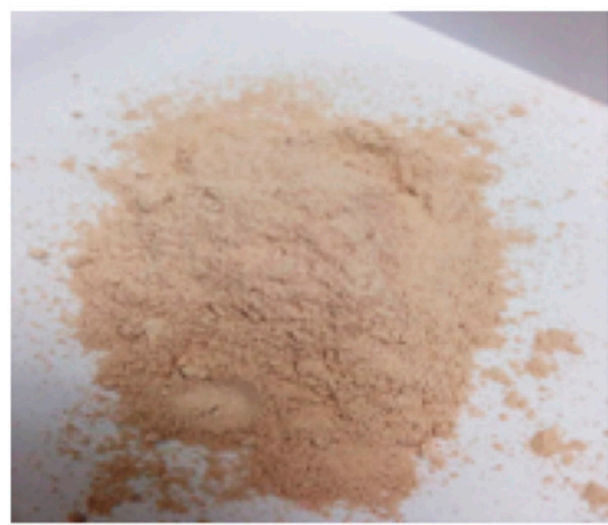

(B)

Figure 5. Appearance of albendazole self-microemulsifying chewable tablets: (A) microemulsion absorbed from colloidal silica and (B) albendazole self-microemulsifying granules.

The flow characteristic of the SMEDDS formulations in terms of angle of repose was determined. In all SMEDDS formulations, flow properties increased when incorporating the self-microemulsion system (F4). The values for angle of repose of less than $40^{\circ}$ yields a fair powder flow requiring no aid, and less than $35^{\circ}$ represents good flow properties [23]. Flow properties based on angle of repose values were observed in the order of F2 $\left(31.1^{\circ}\right)>\mathrm{F} 1\left(32.4^{\circ}\right)>\mathrm{F} 3\left(33.2^{\circ}\right)>\mathrm{F} 4\left(34.1^{\circ}\right)$ as shown in Table 3 , indicating good free-flowing properties in all samples [23].

\subsection{Physical and Chemical Characteristics of Albendazole Self-Microemulsion Chewable Granules and Tablets}

Albendazole raw material, used as a model drug in this study, is a crystalline solid with irregular shape [26]. Powder X-ray diffraction (PXRD) was performed to identify the crystalline state of albendazole raw material and albendazole self-microemulsifying chewable tablets. The X-ray diffractogram of albendazole in the formulation is shown in Figure 6. Albendazole raw material showed numerous sharp and intense peaks at diffraction angles of $7.18^{\circ}, 11.28^{\circ}, 17.93^{\circ}, 19.48^{\circ}, 20.78^{\circ}$, $25.33^{\circ}$, and $27.68^{\circ}$, indicating its high crystallinity. The XRD patterns of SMEDDS formulations F1-F2 show sharp and intense crystalline peaks, indicating that the physical state of albendazole, in the formulations, remain crystalline in the SMEDDS formulations. In the same results, formulation F3, 
a physical mixture of albendazole with SMEDDS excipients, shows a crystalline structure, as suggested by the XRD diffraction patterns, similar to the formulations, F1 and F2. The major peaks of these samples are similar to those obtained in the SMEDDS samples, in terms of intensity and position. The last formulation, F4, was prepared by the conventional wet granulation method without SMEDDS excipients. It showed very high intensity diffraction peaks, probably due to the absence of oil and surfactants with partially dissolved albendazole. Albendazole did not transform from crystalline to an amorphous state, as all the formulations used a high portion of pure albendazole raw material.

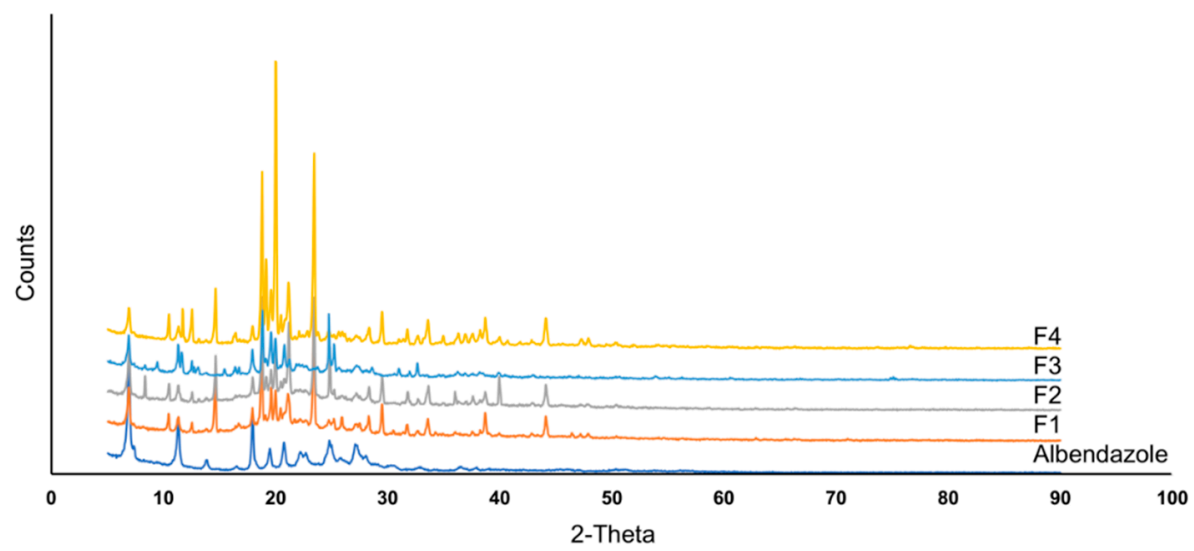

Figure 6. X-ray diffractogram of albendazole and albendazole self-microemulsifying chewable tablets formulation F1-F4.

The results obtained from IR studies showed no interaction between the drug and other excipients used in the formulation. FT-IR of albendazole showed intense bands at 1095.6, 1268.8, 1588.7, $1632.1,1713.8$, and $3318.6 \mathrm{~cm}^{-1}$, corresponding to the presence of functional groups such as aromatic compounds, carbonyl, alkyl, and amine. The FT-IR of albendazole formulations F1-F4 showed intense bands at the same wave number, indicating no change in the functional groups confirming undisturbed structure of albendazole, and suggesting that there was no drug-excipient interaction (Figure 7).

F1-F4 formulations were evaluated for all physical parameters such as weight variation, diameter, thickness, hardness, and friability (Table 4). Thickness ranged from $5.64 \pm 0.02 \mathrm{~mm}$ to $5.72 \pm 0.06 \mathrm{~mm}$, due to the different composition of tablets and granules, characteristic of each formulation. Weight variation and friability were found to be within United State Pharmacopoeia (USP) specifications [23]. Percent weight variation was well within the acceptable limit for uncoated tablets, as per USP specifications. Tablets with the greatest hardness show longer disintegration time, and since mechanical integrity is of paramount importance in the successful formulation of tablets, the hardness of tablets was determined. The friability of albendazole SMEDDS was less than $1 \%$, which is acceptable according to USP criteria. None of the 10 tablets tested were outside the range of $85-115 \%$ of the dosage claimed on their commercial label. These results indicate that the dosage form had uniform distribution and proper dose of the active ingredient. The disintegration time was less than 3 min for all compositions, indicating that they can be used for formulation as chewable tablets. We also found that hardness had no significant effect on drug release, although low hardness decreased the disintegration time. However, since the friability of the tablets was compromised to avoid breaking or erosion, a narrow range of hardness, between 35-45 N, was selected for the compression of batches. Mean values with standard deviation of all physical parameters and drug content for all formulations are shown in Table 3. Drug content of albendazole-loaded SMEDDS preparations are listed in Table 3. In general, the drug content of active ingredients was set within the limit of $90-110 \%$ of the labeled claim [23]. All SMEDDS formulations showed drug content in the range of 100-101\%, indicating that the albendazole-containing SMEDDS was sufficiently adsorbed onto the solid carriers, whilst drug content was constant, indicating that the manufacturing process did not destroy albendazole during preparation. 

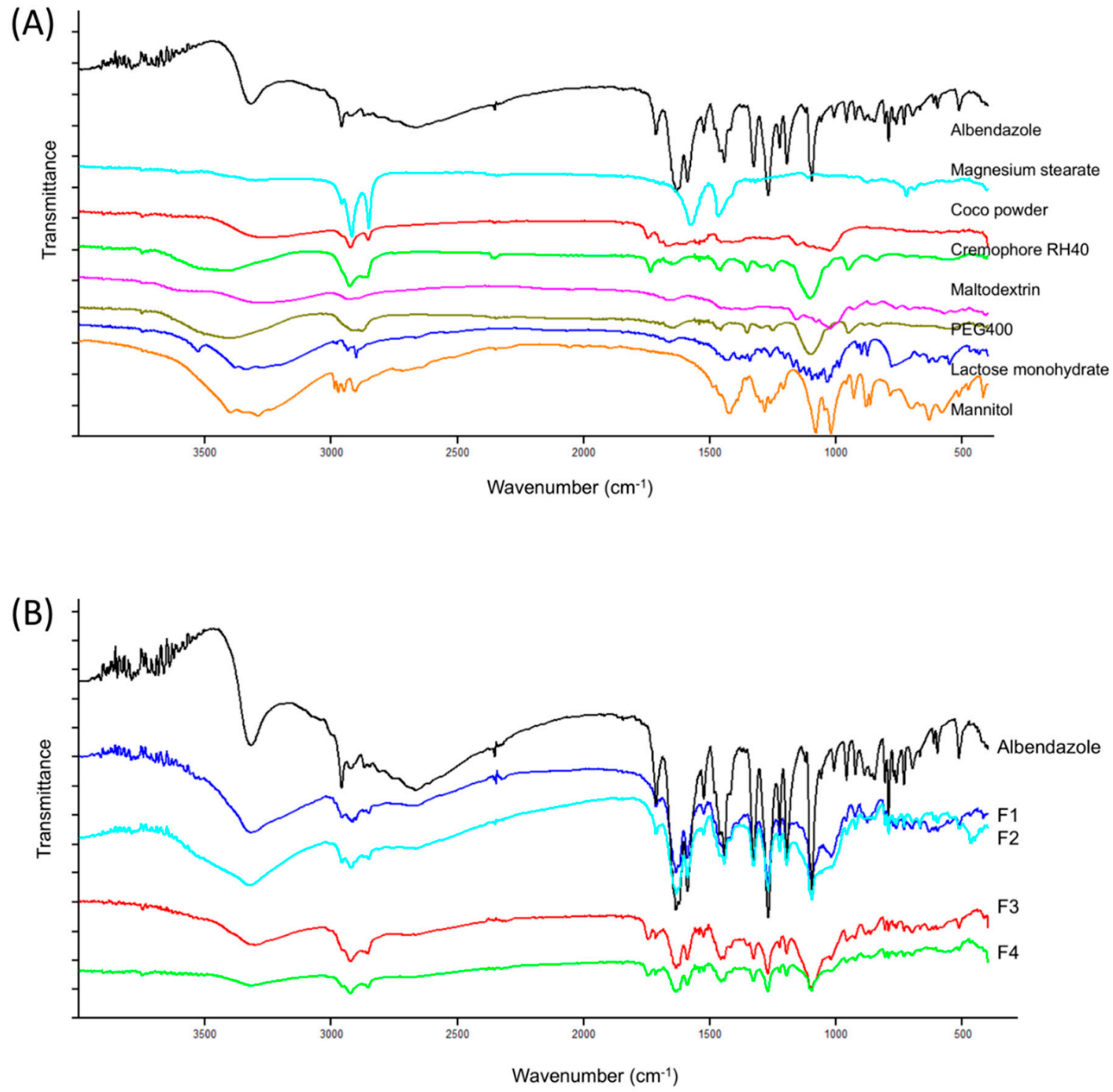

Figure 7. Fourier transfrom infrared (FT-IR) spectrum of (A) albendazole and excipients used in the formulation (B) albendazole and albendazole SMEDDS chewable tablets formulation F1-F4.

Table 4. Properties and assay results for the albendazole SMEDDS chewable granules and tablets $(\mathrm{F} 1-\mathrm{F} 4)($ mean $\pm \mathrm{SD}, n=10)$.

\begin{tabular}{ccccccc}
\hline Formulation & $\begin{array}{c}\text { Angle of } \\
\text { Repose } \mathbf{(}^{\circ}\end{array}$ & $\begin{array}{c}\text { Thickness } \\
(\mathbf{m m})\end{array}$ & $\begin{array}{c}\text { Hardness } \\
\mathbf{( N )}\end{array}$ & $\begin{array}{c}\text { Friability } \\
\mathbf{( \% )}\end{array}$ & $\begin{array}{c}\text { Disintegration } \\
\text { Time (min) }\end{array}$ & \%LA \\
\hline F1 & $32.4 \pm 0.02$ & $5.65 \pm 0.05$ & $38.2 \pm 11.8$ & $0.45 \pm 0.21$ & $<3$ & $101.21 \pm 0.23$ \\
F2 & $31.1 \pm 0.03$ & $5.64 \pm 0.02$ & $37.2 \pm 7.8$ & $0.23 \pm 0.11$ & $<3$ & $100.46 \pm 1.11$ \\
F3 & $33.2 \pm 0.01$ & $5.71 \pm 0.04$ & $40.2 \pm 4.9$ & $0.16 \pm 0.57$ & $<3$ & $100.15 \pm 0.59$ \\
F4 & $34.1 \pm 0.03$ & $5.72 \pm 0.06$ & $41.2 \pm 2.0$ & $0.89 \pm 0.28$ & $<3$ & $101.05 \pm 0.03$ \\
\hline
\end{tabular}

\subsection{In Vitro Dissolution of Albendazole SMEDDS Chewable Tablets}

The cumulative percent of drug dissolved, as a function of time, from albendazole self-microemulsion chewable tablets is illustrated in Figure 8. Dissolution from various samples of F1-F4 was observed in a simulated gastric medium $(0.1 \mathrm{~N} \mathrm{HCl}$ medium, $\mathrm{pH} 1.2)$. All the prepared albendazole self-microemulsion chewable tablets (F1 and F2) yielded significantly higher dissolution than the market product, within the 60-min timeframe. The commercial product in this study used Zentel $^{\mathrm{TM}}$ (Glaxosmithkline Pharmaceuticals Ltd., Brentford, UK). The composition of Zentel ${ }^{\mathrm{TM}}$ consists 
of lactose, microcrystalline cellulose, maize starch, croscarmellose sodium, povidone, sodium lauryl sulphate, sunset yellow lake, sodium saccharin, magnesium stearate, orange flavor, vanilla flavor, and passion fruit flavor [44]. We believe that sodium lauryl sulphate, as a surfactant in the commercial products, may enhance dissolution of albendazole that higher than formulation F3 and F4. Although formulation F3 consists of ingredients similar to the formulation F2, it is prepared by a physical mixing method. Albendazole in F3 does not dissolve to form a microemulsion before the formulation of the tablets.

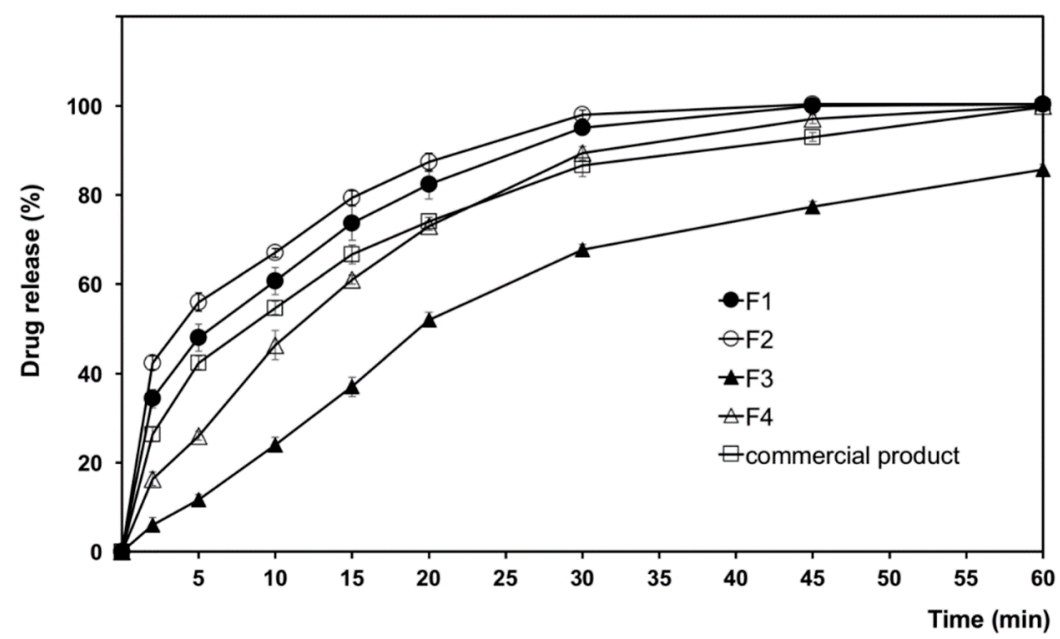

Figure 8. Dissolution profiles of albendazole SMEDDS chewable tablets (F1-F4) and a commercial albendazole tablet in $0.1 \mathrm{~N} \mathrm{HCl}$ medium $\mathrm{pH} 1.2$.

The percentage of drug dissolved from test tablets in $60 \mathrm{~min}$ can be arranged in descending order as follows: F2 $>$ F1 $>$ market product $>$ F4 $>$ F3, indicating that albendazole self-microemulsion chewable tablets enhanced the dissolution of albendazole. Complete dissolution was achieved from F3 in 30 min. Decrease in the dissolution rate in case of F4 as the formulation did not contain a microemulsion system. In contrast, dissolution of formulation F3 decreased due to the high content of oil, which was not formulated as a self-microemulsion system.

Albendazole dissolution significantly increased to over $20 \%$ for the same time period as compared with the formulation without a self-microemulsion system. There was a significant increase in albendazole dissolution in the SMEDDS formulations as compared to the commercial product. The dissolution rates of F2 was similar to F1, due to the complete solubilization of the albendazole in the microemulsion system. SMEDDS improved the drug release rate as compared to the conventional albendazole tablets (F4) or commercial tablets because the free energy required to form a microemulsion is very low and the spontaneous formation of an interface between water and oil droplets is possible $[29,45]$.

Both self-microemulsion systems F1 and F2 showed high dissolution rates ( $>90 \%$ in simulated gastric medium, $\mathrm{pH} 1.2$ for $1 \mathrm{~h}$ ). F2 was selected for further in vivo pharmacokinetics studies, since it dissolved somewhat faster, and due to its overall superior performance. Therefore, it was subjected to further in vivo studies for comparison with the commercial compressed chewable tablets.

\subsection{Stability Studies}

After six months at accelerated storage at $40{ }^{\circ} \mathrm{C} / 75 \% \mathrm{RH}$, storage at $30{ }^{\circ} \mathrm{C} / 75 \% \mathrm{RH}$, or storage at room temperature and at ambient humidity, there were few differences in appearance, disintegration time, friability and drug content before and after the storage period (Table 5). At $30 \pm 2{ }^{\circ} \mathrm{C} / 75 \pm 5 \%$ relative humidity, hardness was slightly increased, likely due to the absorbed moisture. This indicates that the formulation was fairly stable at both storage conditions. 
Table 5. Of the albendazole self-microemulsifying chewable tablet (F2) stored at $30{ }^{\circ} \mathrm{C} / 75 \%$ relative humidity $(\mathrm{RH})$ and $40{ }^{\circ} \mathrm{C} / 75 \% \mathrm{RH}$ (mean $\left.\pm \mathrm{SD}, n=10\right)$.

\begin{tabular}{|c|c|c|c|c|}
\hline \multirow{2}{*}{$\begin{array}{c}\text { Storage } \\
\text { Conditions }\end{array}$} & \multirow{2}{*}{ Test } & \multicolumn{3}{|c|}{ Storage Period } \\
\hline & & 1 Month & 3 Months & 6 Months \\
\hline \multirow{6}{*}{ Room temperature } & Appearance & \multicolumn{3}{|c|}{ Light brown, round, flat tablet } \\
\hline & Hardness (N) & $38.2 \pm 10.8^{\circ}$ & $37.2 \pm 4.8$ & $38.2 \pm 6.9$ \\
\hline & Disintegration time (min) & $<3$ & $<3$ & $<3$ \\
\hline & Friability $(\%)$ & $<1$ & $<1$ & $<1$ \\
\hline & Water content (\%) & $3.32 \pm 0.13$ & $3.45 \pm 0.82$ & $3.51 \pm 0.88$ \\
\hline & Drug content $(\%)$ & $100.16 \pm 0.64$ & $99.26 \pm 1.67$ & $100.92 \pm 1.32$ \\
\hline \multirow{6}{*}{$30^{\circ} \mathrm{C} / 75 \% \mathrm{RH}$} & Appearance & \multicolumn{3}{|c|}{ Light brown, round, flat tablet } \\
\hline & Hardness (N) & $37.2 \pm 11.8$ & $38.2 \pm 4.9$ & $41.2 \pm 2.0$ \\
\hline & Disintegration time (min) & $<3$ & $<3$ & $<3$ \\
\hline & Friability (\%) & $<1$ & $<1$ & $<1$ \\
\hline & Water content (\%) & $3.12 \pm 0.53$ & $3.65 \pm 1.02$ & $4.01 \pm 1.12$ \\
\hline & Drug content $(\%)$ & $101.03 \pm 1.14$ & $101.26 \pm 0.87$ & $101.39 \pm 0.23$ \\
\hline \multirow{6}{*}{$40^{\circ} \mathrm{C} / 75 \% \mathrm{RH}$} & Appearance & \multicolumn{3}{|c|}{ Light brown, round, flat tablet } \\
\hline & Hardness (N) & $38.1 \pm 1.9$ & $38.2 \pm 4.8$ & $41.3 \pm 2.0$ \\
\hline & Disintegration time (min) & $<3$ & $<3$ & $<3$ \\
\hline & Friability $(\%)$ & $<1$ & $<1$ & $<1$ \\
\hline & Water content (\%) & $3.00 \pm 0.12$ & $3.22 \pm 0.65$ & $3.41 \pm 0.29$ \\
\hline & Drug content (\%) & $101.05 \pm 0.57$ & $100.69 \pm 1.28$ & $99.65 \pm 1.56$ \\
\hline
\end{tabular}

The water contents were calculated according to the following equation:

$$
\text { water content }(\%)=\left[W_{\mathrm{t}} / W_{0}\right] \times 100
$$

where, $W_{0}$ and $W_{t}$ are the water content in each sample at time 0 and time $t$, respectively.

Albendazole self-microemulsion chewable tablet, F2, showed no significant drug loss, suggesting that it was the most stable among the formulations tested in this study. Meanwhile, water content slightly increased under all storage conditions, even though there were no significant differences and tablets were not hygroscopic. This is likely due to PVC serving as a poor moisture barrier.

\subsection{Pharmacokinetic Analysis of Albendazole SMEDDS Chewable Tablets}

Figure 9 shows the change in the mean plasma concentration of albendazole after the oral administration of the F2 albendazole self-microemulsion chewable tablet, albendazole commercial chewable tablets, and albendazole powder, at a dose of $50 \mathrm{mg} / \mathrm{kg}$ albendazole, in rats. The albendazole commercial tablets and powder yielded a lower total plasma concentration compared to the F2 chewable tablet formulation. One to $12 \mathrm{~h}$ after dosing, plasma concentrations of both the commercial tablets and albendazole powder were significantly lower compared with the F2 chewable tablet $(p<0.05)$, likely due to their lower drug solubility and dissolution. Some studies indicate that relatively higher plasma concentrations of albendazole with SMEDDS were due to the increase in solubility and dissolution of albendazole from this system $[4,13]$. The pharmacokinetic parameters are shown in Table 6. The albendazole SMEDDS formulations showed significantly higher AUC and $C_{\max }$ compared to the commercial tablets and albendazole powder $(p$-value $<0.05)$ but the AUC and $C_{\max }$ of the commercial tablets and albendazole powder showed bioequivalent ( $p$-value $>0.05)$. In particular, the AUC values of albendazole SMEDDS were about 1.3-fold higher than that of the albendazole powder. However, AUC did not differ between albendazole commercial tablets and albendazole powder. Although $C_{\max }$ values were about 1.2 fold higher as compared to the corresponding values for albendazole powder, $T_{\max }$ and $t_{1 / 2}$ values of both formulations were not significantly different from the commercial powder. Our results suggest that the enhanced oral bioavailability of albendazole 
in the SMEDDS formulation led to a marked increase in drug absorption due to its improved solubility and dissolution, supporting the hypothesis illustrated in Figure 1.

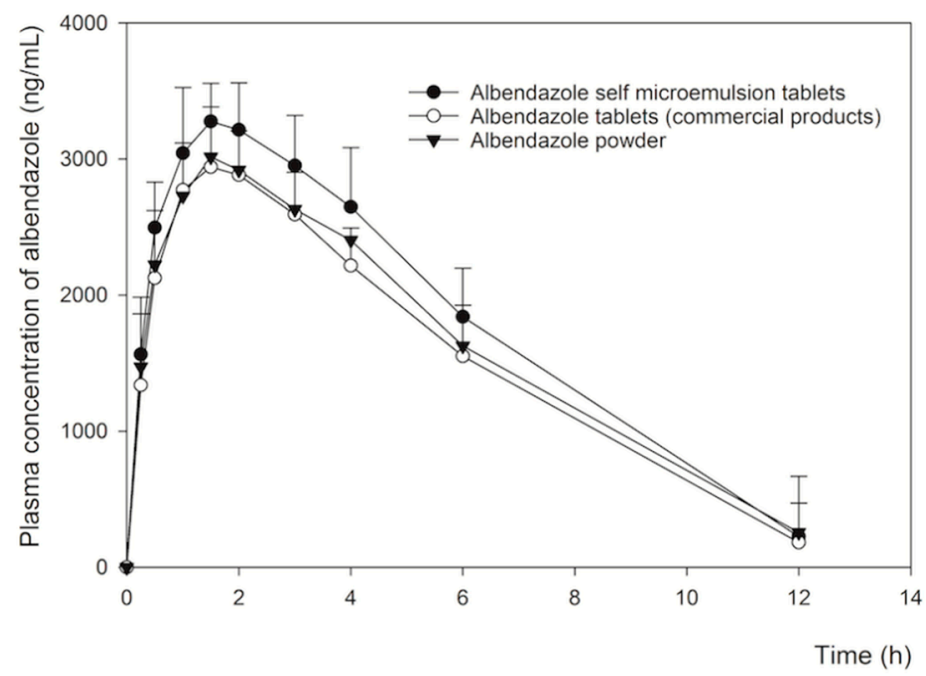

Figure 9. Mean plasma concentration/time profiles of albendazole sulfoxide after oral administration of albendazole at a dose of $50 \mathrm{mg} / \mathrm{kg}$ in SMEDDS chewable tablets formulation F2 (solid circles, $n=5$ ), albendazole commercial tablets (open circles, $n=5$ ) and albendazole powder (solid triangles, $n=5$ ) in rats. Data shown indicate mean \pm standard deviation $(\mathrm{SD})$.

Table 6. Comparative mean (SD) of pharmacokinetic parameters of albendazole sulfoxide in rats after oral administration.

\begin{tabular}{ccccc}
\hline \multirow{2}{*}{$\begin{array}{c}\text { Pharmacokinetic } \\
\text { Parameters }\end{array}$} & \multicolumn{3}{c}{ Albendazole Sulfoxide } & $\begin{array}{c}\text { Relative } \\
\text { Bioavailability (\%) }\end{array}$ \\
\cline { 2 - 4 } & $\begin{array}{c}\text { Self-Microemulsion } \\
\text { Tablets }\end{array}$ & $\begin{array}{c}\text { Commercial } \\
\text { Tablets }\end{array}$ & Powder & $131.2 *$ \\
\hline $\mathrm{AUC}_{0-\infty}(\mathrm{ng} \mathrm{h} / \mathrm{mL})$ & $1982 \pm 785$ & $1502 \pm 357$ & $1591 \pm 482$ & \\
$C_{\max }(\mathrm{ng} / \mathrm{mL})$ & $3460 \pm 235$ & $1875 \pm 123$ & $1911 \pm 198$ & \\
$T_{\max }(\mathrm{h})$ & $1.00 \pm 0.21$ & $1.00 \pm 0.16$ & $1.00 \pm 0.43$ & \\
$t_{1 / 2}(\mathrm{~h})$ & $3.11 \pm 1.21$ & $3.02 \pm 0.86$ & $2.95 \pm 1.02$ & \\
\hline
\end{tabular}

${ }^{*}$ Relative bioavailability with respect to commercial tablets $(p \leq 0.05)$.

\section{Conclusions}

In this study, two formulations of albendazole were developed as SMEDDS based on either sesame oil + PEG400 + Tween80 or soybean oil + Cremophor RH40 + Tween80, in order to increase the solubility, stability, and bioavailability of albendazole. Using this formulation, as a chewable tablet, the dissolution and bioavailability of albendazole improved compared to a commercial formulation and albendazole powder. This tablet was stable under accelerated conditions of $40{ }^{\circ} \mathrm{C}$ and $75 \%$ $\mathrm{RH}$, long-term conditions at $30{ }^{\circ} \mathrm{C}$ and $75 \% \mathrm{RH}$, and room temperature with ambient humidity for six months.

Author Contributions: Conceptualization, S.S. and A.A.; methodology, S.S., A.A., A.S.Y., T.S. (Teerapol Srichana), N.C., T.S. (Tan Suwandecha); writing and original draft preparation, S.S., A.A., A.S.Y., T.S. (Teerapol Srichana), N.C., T.S. (Tan Suwandecha); writing and review and editing, S.S., A.A., T.S. (Teerapol Srichana)

Funding: This research was funded by Walailak University (Grant Number WU60117 and WU60117-2), Drug and Cosmetics Excellence Center, School of Pharmacy, Walailak University.

Acknowledgments: The authors would like to thank the pharmacy students Peemmarat Poolsuk, Pheemagorn Punnit, and Suphalak Homchuam who helped in the experiments during a pharmacy senior project.

Conflicts of Interest: The authors declare no conflict of interest. 


\section{References}

1. Hong, S. Albendazole and praziquantel: Review and safety mornitoring in Korea. Infect. Chemother. 2018, 50, 1-10. [CrossRef]

2. Dayan, A.D. Albendazole, mebendzole and praziquantel. Review of non-clinical toxicity and pharmacokinetics. Acta Trop. 2003, 86, 141-159. [CrossRef]

3. Horton, J. Albendazole: A review of anthelmintic efficacy and safety in humans. Parasitology 2000, 121, S113-S132. [CrossRef] [PubMed]

4. Meena, A.K.; Sharma, K.; Kandaswamy, M.; Rajagopal, S.; Mullangi, R. Formulation development of an albendazole self-emulsifying drug delivery system (SEDDS) with enhanced systemic exposure. Acta Pharm. 2012, 62, 563-580. [CrossRef] [PubMed]

5. Kawabata, Y.; Wada, K.; Nakatani, M.; Yamada, S.; Onoue, S. Formulation design for poorly water-soluble drugs based on biopharmaceutics classification system: Basic approaches and practical applications. Int. J. Pharm. 2011, 420, 1-10. [CrossRef] [PubMed]

6. Rigter, I.M.; Schipper, H.G.; Koopmans, R.P.; Kan, H.J.M.; Frijlink, H.W.; Kager, P.A.; Guchelaar, H.-J. Relative bioavailability of three newly developed albendazole formulations: A randomized crossover study with healthy volunteers. Antimicrob. Agents Chemother. 2004, 48, 1051-1054. [CrossRef] [PubMed]

7. Sriamornsak, P.; Limmatvapirat, S.; Piriyaprasarth, S.; Mansakmanee, P.; Huang, Z. A new self-emulsifying formulation of mefenamic acid with enhanced drug dissolution. Asian J. Pharm. Sci. 2015, 10, 121-127. [CrossRef]

8. Hirani, J.J.; Rathod, D.A.; Vadalia, K.R. Orally disintegrating tablets: A review. Trop. J. Pharm. Res. 2009, 8, 161-172. [CrossRef]

9. Lee, J.; Kim, H.H.; Cho, Y.H.; Koo, T.; Lee, G.W. Development and evaluation of raloxifene-hydrochlorideloaded supersaturatable SMEDDS containing an acidifier. Pharmaceutics 2018, 10, 78. [CrossRef] [PubMed]

10. Gursoy, R.; Benita, S. Self-emulsifying drug delivery system (SEDDS) for improved oral delivery of lipophilic drugs. Biomed. Pharmacother. 2004, 58, 173-182. [CrossRef]

11. Qiao, J.; Ji, D.; Sun, S.; Zhang, G.; Liu, X.; Sun, B.; Guan, Q. Oral bioavailability and lymphatic transport of pueraria flavone-loaded self-emulsifying drug-delivery systems containing sodium taurocholate in rats. Pharmaceutics 2018, 10, 147. [CrossRef] [PubMed]

12. Solè, I.; Solans, C.; Maestro, A.; González, C.; Gutiérrez, J.M. Study of nano-emulsion formation by dilution of microemulsions. J. Colloid Interface Sci. 2012, 376, 133-139. [CrossRef] [PubMed]

13. Mukherjee, T.; Plakogiannis, F.M. Development and oral bioavailability assessment of a supersaturated self-microemulsifying drug delivery system (SMEDDS) of albendazole. J. Pharm. Pharmacol. 2010, 62, 1112-1120. [CrossRef] [PubMed]

14. Midha, K.; Nagpal, M.; Aggarwal, G.; Singh, T.G. Development of dispersible self-microemulsifying tablet of atorvastatin. Pharm. Methods 2015, 6, 9-25. [CrossRef]

15. Hasan, N.M.Y.; Almalki, D.M.; Althuwaybi, M.J.K.; Alshehri, H.M. SMEDDS tablet: Compatability of solid SMEDDS using various pharmaceutical tablet excipients. Int. J. Pharm. Pharm. Sci. 2016, 8, 246-251. [CrossRef]

16. Midha, K.; Nagpal, M.; Singh, G.; Aggarwal, G. Prospectives of solid self-microemulsifying systems in novel drug delivery. Curr. Drug Deliv. 2017, 14, 1078-1096. [CrossRef]

17. Oh, D.H.; Kang, J.H.; Kim, D.W.; Lee, B.J.; Kim, J.O.; Yong, C.S.; Choi, H.G. Comparison of solid self-microemulsifying drug delivery system (solid SMEDDS) prepared with hydrophilic and hydrophobic solid carrier. Int. J. Pharm. 2012, 420, 412-418. [CrossRef]

18. Qi, X.; Qin, J.; Ma, N.; Chou, X.; Wu, Z. Solid self-microemulsifying dispersible tablets of celastrol: Formulation development, characterization and bioavailability evaluation. Int. J. Pharm. 2014, 472, 40-47. [CrossRef]

19. Seljak, K.B.; Ilic, I.G.; Gasperlin, M.; Pobirk, A.Z. Self-microemulsifying tablets prepared by direct compression for improved T resveratrol delivery. Int. J. Pharm. 2018, 548, 263-275. [CrossRef]

20. Rathod, D.M.; Patel, K.R.; Mistri, H.N.; Jangid, A.G.; Shrivastav, P.S.; Sanyal, M. Liquid chromatography-tandem mass spectrometry method for simultaneous determination of albendazole and albendazole sulfoxide in human plasma for bioequivalence studies. J. Pharm. Anal. 2016, 6, 226-234. [CrossRef] 
21. Valois, M.E.C.; Takayanagui, O.M.; Bonato, P.S.; Lanchote, V.L.; Carvalho, D. Determination of albendazole metabolites in plasma by HPLC. J. Anal. Toxicol. 1994, 18, 86-90. [CrossRef] [PubMed]

22. Wu, Z.; Medlicott, N.J.; Razzak, M.; Tucker, I.G. Development and optimization of a rapid HPLC method for analysis of ribobendazole and albendazole sulfone in sheep plasma. J. Pharm. Biomed. Anal. 2005, 39, 225-232. [CrossRef] [PubMed]

23. United States Pharmacopeial Convention. USP36 NF31, US Pharmacopoeia National Formulary; United States Pharmacopeial Convention: North Bethesda, MD, USA, 2012.

24. Hanmant, B.S.; Karmude, K.T.; Gurav, Y.A.; Sandeep, W. Formulation, optimization and development of albendazole tablet for the improvement of oral bioavailability. World J. Pharm. Res. 2016, 5, 1170-1181.

25. Anusha, V.; Palanichamy, S.; Sugumar, M.; Rajesh, M.; Parasakthi, N.; Das, T.G.R.; Ramasubramaniyan, P.; Thirupathi, T. Formulation and characterization of albendazole chewable tablets. Der Pharmacia Sinica 2012, 3, 211-216.

26. Prashant, R.; Rakesh, M.; Tanaji, N.; Sushikumar, P. Solubility and dissolution enhancement of albendazole by spherical crystallization. Asian J. Biomed. Pharm. Sci. 2016, 6, 9-14. [CrossRef]

27. ASEAN Guideline on Stability of Drug Products. May 2013. Available online: http://www.hsa.gov. sg/content/dam/HSA/HPRG/Western_Medicine/Overview_Framework_Policies/Guidelines_on_ Drug_Registration/ASEAN\%20STABILITY\%20GUIDELINE\%20(version\%206.0).pdf (accessed on 15 November 2017).

28. Bajaj, S.; Singla, D.; Sakhuja, N. Stability testing of pharmaceutical products. J. Appl. Pharm. Sci. 2012, 2, 129-138.

29. Kim, D.W.; Kwon, M.S.; Yousaf, A.M.; Balakrishnan, P.; Park, J.H.; Kim, D.S.; Lee, B.; Park, Y.J.; Yong, C.S.; Kim, J.O.; et al. Comparison of a solid SMEDDS and solid dispersion for enhanced stability and bioavailability of clopidogrel napadisilate. Carbohydr. Polym. 2014, 114, 365-374. [CrossRef]

30. Kang, J.H.; Oh, D.H.; Oh, Y.K.; Yong, C.S.; Choi, H.G. Effects of solid carriers on the crystalline properties, dissolution and bioavailability of flurbiprofen in solid self-nanoemulsifying drug delivery system (solid SNEDDS). Eur. J. Pharm. Biopharm. 2012, 80, 289-297. [CrossRef]

31. Tran, T.; Rades, T.; Müllertz, A. Formulation of self-nanoemulsifying drug delivery systems containg monoacyl phosphatidylcholine and Kolliphar ${ }^{\circledR}$ RH40 using experimental design. Asian J. Pharm. Sci. 2018, 13, 536-545. [CrossRef]

32. Jyothi, B.J.; Sreelakshmi, K. Design and evaluation of self-nanoemulsifying drug delivery system of flutamide. J. Young Pharm. 2011, 3, 4-8. [CrossRef]

33. Köller, S.; Nardin, I.; Markt, R.; Griesser, J.; Prüfert, F.; Bernkop-Schnürch, A. Self-emulsifying drug delivery systems: Design of a novel vaginal delivery systems for curcumin. Eur. J. Pharm. Biopharm. 2017, 115, 268-275. [CrossRef] [PubMed]

34. Pouton, C.W.; Porter, C.J.H. Formulation of lipid-based delivery systems for oral administration: Materials, methods and strategies. Adv. Drug Deliv. Rev. 2008, 60, 625-637. [CrossRef] [PubMed]

35. Shao, A.; Chen, G.; Jiang, N.; Li, Y.; Zhang, X.; Wen, L.; Yang, F.; Wei, S. Development and evaluation of self-microemulsifying liquid and granule formulations of Brucea javanica oil. Arch. Pharm. Res. 2013, 36, 993-1003. [CrossRef] [PubMed]

36. Yeom, D.W.; Son, H.Y.; Kim, J.H.; Kim, S.R.; Lee, S.G.; Song, S.H.; Chae, B.R.; Choi, Y.W. Development of a solidified self-microemulsifying drug delivery system (S-SMEDDS) for atorvastatin calcium with improved dissolution and bioavailability. Int. J. Pharm. 2016, 506, 302-311. [CrossRef] [PubMed]

37. Nazzal, S.; Smalyukh, I.I.; Lavrentovich, O.D.; Khan, M.A. Preparation and in vitro characterization of a eutectic based semisolid self-nanoemulsified drug delivery system (SNEDDS) of ubiquinone: Mechanism and progress of emulsion formation. Int. J. Pharm. 2002, 235, 247-265. [CrossRef]

38. Van Speybroeck, M.; Williams, H.D.; Nguyen, T.H.; Anby, M.U.; Porter, C.J.; Augustijns, P. Incomplete desorption of liquid excipients reduces the in vitro and in vivo performance of self-emulsifying drug delivery systems solidified by adsorption onto an inorganic mesoporous carrier. Mol. Pharm. 2012, 9, 2750-2760. [CrossRef] [PubMed]

39. Christensen, K.L.; Pedersen, G.P.; Kristensen, H.G. Physical stability of redispersible dry emulsions containing amorphous sucrose. Eur. J. Pharm. Biopharm. 2002, 53, 147-153. [CrossRef]

40. Pedersen, G.P.; Fäldt, P.; Bergenståhl, B.; Kristensen, H.G. Solid state characterisation of a dry emulsion: A potential drug delivery system. Int. J. Pharm. 1998, 171, 257-270. [CrossRef] 
41. Sawatdee, S.; Atipairin, A.; Sae Yoon, A.; Srichana, T.; Changsan, N. Enhanced dissolution of sildenafil citrate as dry foam tablets. Pharm. Dev. Technol. 2019, 24,1-11. [CrossRef]

42. Zaki, A.; Anwa, E.; Surini, S. Formulation of a fast-disintegrating tablet using maltodextrin DE10-15 and pregelatinizated cassava atarch as excipients. Int. J. App. Pharm. 2017, 9 (Suppl. 1), 71-73. [CrossRef]

43. Parikh, A.; Agarwal, S.; Raut, K. A review on applications of maltodextrin in pharmaceutical industry. Int. J. Pharm. Bio. Sci. 2014, 4, 67-74.

44. Package Insert of Zentel ${ }^{\mathrm{TM}}$ (Albendazole). Available online: https://sg.gsk.com/media/574582/zentel.pdf (accessed on 10 March 2019).

45. Balakrishnan, P.; Lee, B.J.; Oh, D.H.; Kim, J.O.; Hong, M.J.; Jee, J.P.; Kim, J.A.; Yoo, B.K.; Woo, J.S.; Yong, C.S.; et al. Enhanced oral bioavailability of dexibuprofen by a novel solid self-emulsifying drug delivery system (SEDDS). Eur. J. Pharm. Biopharm. 2009, 72, 539-545. [CrossRef]

2019 by the authors. Licensee MDPI, Basel, Switzerland. This article is an open access article distributed under the terms and conditions of the Creative Commons Attribution (CC BY) license (http:/ / creativecommons.org/licenses/by/4.0/). 